\title{
Dynamical System of Three Magnetic Layers in the Presence of Porous Media
}

\author{
Sameh A. Alkharashi ${ }^{1,2}$ \\ ${ }^{1}$ Quesna Technical College, Tanta Technical Commercial Institute, Ministry of Higher Education, Cairo, Egypt \\ ${ }^{2}$ Applied Sciences Department, College of Technological Studies, The Public Authority for Applied Education \\ and Training (PAAET), Adiliya, Kuwait \\ Email: sameh7977@yahoo.com
}

Received 2 January 2015; accepted 5 February 2015; published 23 March 2015

Copyright @ 2015 by author and Scientific Research Publishing Inc. This work is licensed under the Creative Commons Attribution International License (CC BY). http://creativecommons.org/licenses/by/4.0/

\section{Abstract}

This paper concerns the linear stability of three viscous fluid layers in porous media. The system is composed of a middle fluid embedded between two semi-infinite fluids, in which the effect of the normal magnetic field is to introduce. The principle aim of this work is to investigate the influence of fluid viscosity and the porosity effect on the growth rate in the presence of normal magnetic field. The parameters governing the layers flow system, the magnetic properties and porosity effects strongly influence the wave forms and their amplitudes and hence the stability of the fluid. The stability criteria are discussed theoretically and numerically and stability diagrams are obtained, where regions of stability and instability are identified. It is found that the stabilizing role for the magnetic field is retarded when the flow is in porous media. Moreover, the increase in the values of permeability parameters plays a dual role, in stability behavior. It has been found that the phenomenon of the dual (to be either stabilizing or destabilizing) role is found for increasing the permeability parameter. It is established that both the viscosity coefficient and the magnetic permeability damps the growth rate, introducing stabilizing influence. The role of the magnetic field and Reynolds number is to increase the amplitude of the disturbance leading to the destabilization state of the flow system, promote the oscillatory behavior. Influence of the various parameters of the problem on the interface stability is thoroughly discussed.

\section{Keywords}

Viscous Fluids, Magnetic Field, Three Layers Stability, Porous Media

\section{Introduction}

There has been a great deal of interest in magnetic fluids of the stability of hydrodynamic stability. A magnetic 
fluid, also known in the literature as a ferromagnetic fluid or simply a ferrofluid. In many previous researches has shown the importance of studying the hydrodynamic stability problems, for example capillary-gravity waves of permanent form at the interface between two unbounded magnetic fluids in porous media are investigated in paper [1]. In which the system is influenced by the horizontal direction of the magnetic field to the separation face of two semi-infinite homogeneous and incompressible fluids. It is found that the stabilizing role for the magnetic field is retarded when the flow is in porous media. In paper [2] the influence of viscosity on the stability of the plane interface separating two incompressible superposed fluids of uniform densities, when the whole system is immersed in a uniform horizontal magnetic field, has been studied. The authors in the research [3] have carried out the instability of viscous potential flow in a horizontal rectangular channel. The analysis leads to an explicit dispersion relation in which the effects of surface tension and viscosity on the normal stress are not neglected but the effect of shear stresses is. The unsteady electrohydrodynamic stability has been investigated by Elhefnawy [4], where the stability analysis has been made of a basic flow of streaming fluids in the presence of an oblique periodic electric field. A series of studies for hydrodynamics stability have also been initiated by Drazin and Reid [5] and Joseph [6].

The authors in paper [7] have investigated the stability properties of time periodically streaming superposed magnetic fluids through porous media under the influence of an oblique alternating magnetic field. Also, Zakaria et al. [8] have analyzed the effect of an externally applied electric field on the stability of a thin fluid film over an inclined porous plane, using linear and non-linear stability analysis in the long wave limit. Wray et al. [9] have investigate the evolution and stability of a wetting viscous fluid layer flowing down the surface of a cylinder, and surrounded by a conductive gas. Liu et al. [10] have studied the instability properties of two-dimensional nonNewtonian liquid sheets moving in an inviscid gaseous environment.

Based on a modified Darcy's law for a viscoelastic fluid, Sirwah [11] has discussed the linear stability analysis of the electrified surface separating two coaxial Oldroyd-B fluid layers confined between two impermeable rigid cylinders in the presence of both interfacial insoluble surfactant and surface charge through porous media. Also, Tan and Masuoka [12] have extended Stokes? first problem to that for an Oldroyd-B fluid in a porous half space, where an exact solution was obtained by using Fourier sine transform. Zakaria [13] has investigated the time evolution of superposed layers of fluid flowing down inside an inclined permeable channel. Using the Kármán-Pohlhausen approximation, the problem is reduced to the study of the evolution equation for the liquid? liquid interface of the liquids film derived through a long wave approximation. Khan et al. [14] have demonstrated the analytical solutions for the magnetohydrodynamic flow of an Oldroyd-B fluid through a porous medium. They obtained the expressions for the velocity field and the tangential stress by means of the Fourier sine transform. Kumar and Singh [15] have investigated the stability of a plane interface separating two viscoelastic (Rivlin-Ericksen) superposed fluids in the presence of suspended particles.

In the present work we wish to consider an investigation of stability for flow in a porous medium under the effect of a magnetic field that is normal to the interface between the fluids. The considered system is composed of a viscous fluid layer of finite thickness embedded between two semi-infinite fluids. We have concentrated in this work to investigate the mechanisms of stability of three porous layers of fluids in the presence of normal magnetic field. This paper is organized as follows: This section has presented the motivation for the investigation in addition to relevant background information. In next section, we will give a formulation of the problem statement, including the basic equations of the fluid mechanics and Maxwell's equations governing the motion of our model. In the third section and its subsections, are concerned with the derivation of the characteristic equation and numerical estimations for stability configuration. The salient results of our analysis are discussed and some important conclusions are drawn in final section of this paper.

\section{Formulation of the Problem}

The system under consideration is composed of an infinite horizontal viscous fluid sheet of vertical height $2 L$ confined between two semi-infinite superposed incompressible viscous fluids. The fluids are considered to be influenced by the gravity force in the negative $y$-direction. The $x$-axis of the co-ordinate system is parallel to the direction of the fluid sheet flow, and the $y$-axis is normal to the fluid sheet with its origin located at the middle plane of the fluid sheet as shown in Figure 1. The superscripts (1), (2) and (3) refer to the lower fluid, middle sheet and upper fluid respectively, where $\rho^{(r)},(r=1,2,3)$ is the fluid densities, $\mu^{(r)}$ is the magnetic permeability and $\eta^{(r)}$ represents the viscosities of the fluids. The fluids are subjected to external magnetic field 


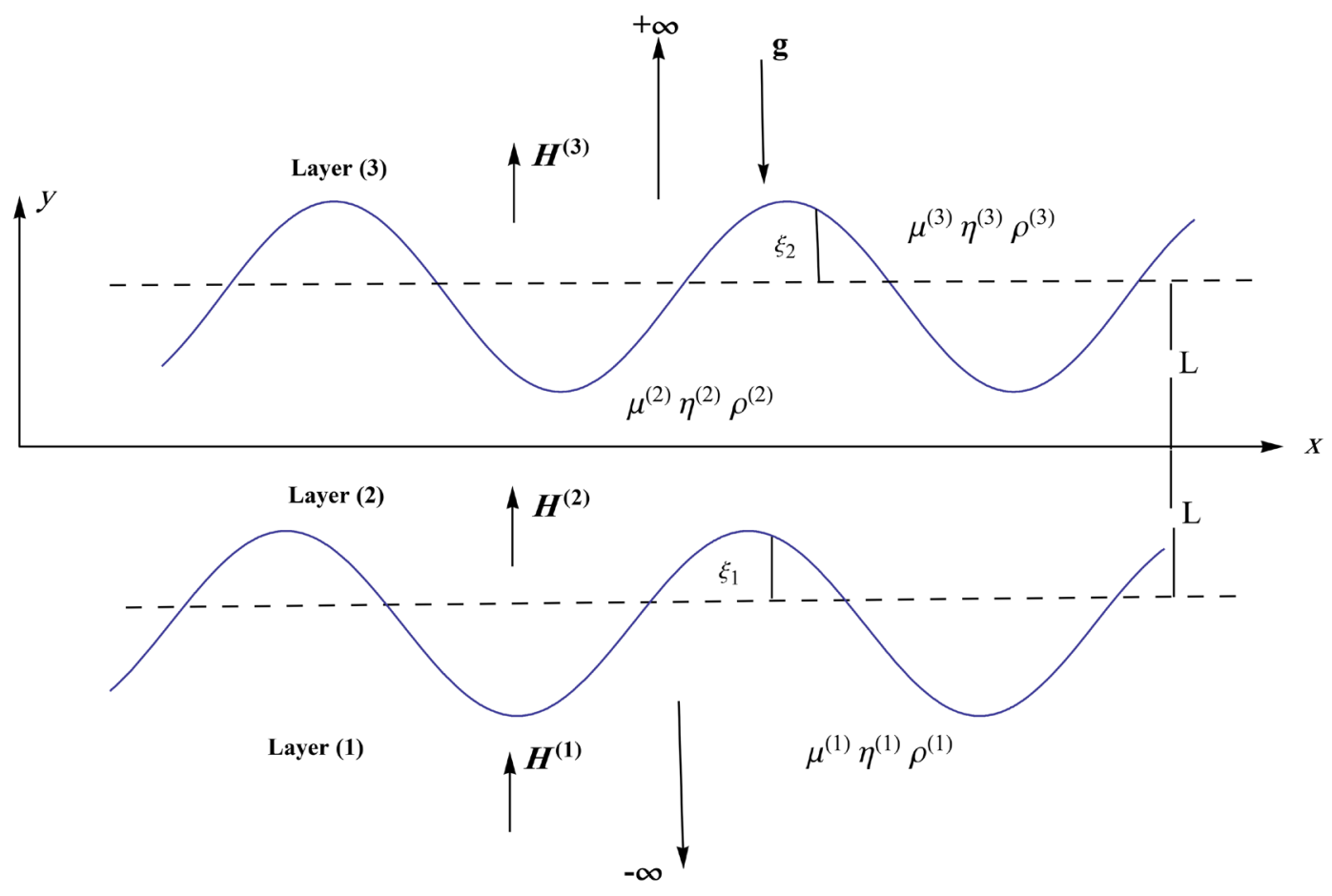

Figure 1. Sketch for the system under consideration. The $y$-axis is taken vertically upwards, the $x$-axis is taken horizontally at the half of the middle sheet.

acting in the positive $y$-direction. The two interfaces are parallel and the flow in each phase is every where parallel to each other. The surface deflections are expressed by $y=\xi_{1}(x, t)$ at $y=-L$ and $y=\xi_{2}(x, t)$ at $y=L$, where at the initial state of the system, we assume that the fluid phases are viscous and have a common flat interfaces at $y=-L$ and $y=L$.

\subsection{Scaling Variables for the Evolution Equations}

First, we will use the dimensionless variables to provide improved insight into the physics and in order to understand hydrodynamic stability better. So we define the corresponding dimensionless variables using the half thickness of the middle fluid sheet $L$ as a length scale. Thus the stream velocity and the time are made dimensionless using $\sqrt{L g}$ and $\sqrt{L / g}$, while the applied magnetic field and the magnetic potential are made dimensionless by $\sqrt{L g \rho^{(2)} / \mu^{(2)}}$ and $L \sqrt{L g \rho^{(2)} / \mu^{(2)}}$, respectively. In addition the viscosity $\rho^{(2)} \sqrt{L^{2} g}$, the pressure $L g \rho_{2}$, the stream function $\sqrt{L^{3} g}$ and the permeability of the porous medium $L^{2} Q$. Furthermore, in the equations of motion, we use the symbols: the magnetic permeability ratio $\hat{\mu}^{(r)}=\mu^{(r)} / \mu^{(2)}$ the fluid density ratio $\hat{\rho}^{(r)}=\rho^{(r)} / \rho^{(2)}$ the dynamic viscosities ratio $\hat{\eta}^{(r)}=\eta^{(r)} / \eta^{(2)}$. Also the Weber number $W_{j}=T_{j} / L^{2} g \rho^{(2)}$, $(j=1,2)$, where $T_{j}$ is the surface tension coefficient.

The dynamics of the problem are described by the simultaneous solution of three field equations: Maxwell's equations, Navier-Stokes equation, and the continuity equation. Assuming a quiescent initial state, therefore the base state velocity in the fluid layers is zero in which the flow is steady and fully developed. Fluid flow through a porous medium is often given by the phenomenological Darcy's equation. Thus, the equations governing twodimensional motion of a viscous incompressible fluid through porous medium are [3]-[7]

$$
\hat{\rho}^{(r)} R_{e}\left(\frac{\partial \mathbf{V}^{(r)}}{\partial t}+\mathbf{V}^{(r)} \cdot \nabla \mathbf{V}^{(r)}\right)=-R_{e} \nabla P^{(r)}+\hat{\eta}^{(r)} \nabla^{2} \mathbf{V}^{(r)}+\hat{\eta}^{(r)} \frac{\mathbf{V}^{(r)}}{Q^{(r)}}
$$

and the equation of continuity will be

$$
\nabla \cdot \mathbf{V}^{(r)}=0
$$


where $R_{e}=\rho^{(2)} \sqrt{d^{3} g} / \eta^{(2)}$ denotes the Reynolds number of the middle layer and $Q^{(r)}$ represents the permeability parameter and $P^{(r)}=p^{(r)}+\rho^{(r)} g y$ is the total hydrostatic pressure and $\nabla \equiv\left(\frac{\partial}{\partial x}, \frac{\partial}{\partial y}\right)$ is the horizontal gradient operator.

In writing Maxwell's equations for the problem, we supposed that the electro-quasi-static approximation is valid for the problem, and hence the magnetic field equations read

$$
\nabla \cdot\left(\mu^{(r)} \mathbf{H}^{(r)}\right)=0 \text { and } \nabla \times \mathbf{H}^{(r)}=\mathbf{0}
$$

Here, $\mathbf{H}^{(r)}$ refers to the magnetic field intensity vector and $\mu^{(r)}$ is the magnetic permeability. The construction of a potential function $\chi^{(r)}$, can be representable as the gradient of the scalar potential such that

$$
\mathbf{H}^{(r)}=-\frac{\partial \chi^{(r)}}{\partial x} \mathbf{e}_{1}+\left(H_{0}^{(r)}-\frac{\partial \chi^{(r)}}{\partial y}\right) \mathbf{e}_{2}
$$

and thus we have the Laplace equation in the form

$$
\frac{\partial^{2} \chi^{(r)}}{\partial x^{2}}+\frac{\partial^{2} \chi^{(r)}}{\partial y^{2}}=0
$$

where $\mathbf{e}_{1}$ and $\mathbf{e}_{2}$ are unit vectors in $x$ - and $y$ - directions.

\subsection{Boundary Conditions}

Solution of the equations of motion cited before is accomplished by utilizing the convenient boundary conditions. The flow field solutions of the above governing equations have to satisfy the kinematic and dynamic boundary conditions at the two interfaces, which can be taken as $y \approx \pm 1$. The normal component of the velocity vector in each of the phases of the system is continuous at dividing surface [16] [17]:

$$
\mathbf{n}_{j} \cdot \mathbf{V}^{(j)}=\mathbf{n}_{j} \cdot \mathbf{V}^{(j+1)}=0, \quad y=(-1)^{j}, j=1,2
$$

where $\mathbf{n}_{j}=-\frac{\partial \xi_{j}(x, t)}{\partial x} \mathbf{e}_{1}+\mathbf{e}_{2}$ is the outward normal unit vector to the interfaces. The condition that the interfaces are moving with the fluids lead to

$$
v^{j,(j+1)}+\frac{\partial \xi_{j}}{\partial t}=0, \quad y=(-1)^{j}, j=1,2
$$

In addition the jump in the shearing stresses is zero across the interfaces, this gives

$$
\left\|\hat{\eta}^{(j)}\left\{\frac{\partial u^{(j)}}{\partial y}+\frac{\partial v^{(j)}}{\partial x}\right\}\right\|_{j}^{j+1}=0, \quad y=(-1)^{j}
$$

where, $\mathbf{V}=(u, v)$ is the velocity vector due to disturbances and the notation $\|\cdot\|$ denotes the jump of a quantity across the interfaces.

Furthermore, the Maxwell's conditions on the magnetic field where no free surface charges are present on the interfaces. The continuity of the normal component of the magnetic displacement at the interfaces reads:

$$
\frac{\partial \chi^{(2)}}{\partial y}-\hat{\mu}^{(1)} \frac{\partial \chi^{(1)}}{\partial y}=0 \text { at } y=-1 \text { and } \frac{\partial \chi^{(2)}}{\partial y}-\hat{\mu}^{(2)} \frac{\partial \chi^{(3)}}{\partial y}=0 \text { at } y=1
$$

The tangential component of the magnetic field is zero across the interfaces, this requires that from this equation, we have 


$$
\begin{aligned}
& \frac{\partial \chi^{(2)}}{\partial x}-\frac{\partial \chi^{(1)}}{\partial x}=\left(\hat{\mu}^{(2)}-1\right) H_{0}^{(1)} \frac{\partial \xi_{1}}{\partial x} \text { at } y=-1 \\
& \frac{\partial \chi^{(3)}}{\partial x}-\frac{\partial \chi^{(2)}}{\partial x}=\left(\frac{\hat{\mu}^{(1)}}{\hat{\mu}^{(2)}}-\hat{\mu}^{(1)}\right) H_{0}^{(1)} \frac{\partial \xi_{2}}{\partial x} \text { at } y=1
\end{aligned}
$$

where, we use the zero order from continuity of the normal component of magnetic field to express both $H_{0}^{(2)}$ and $H_{0}^{(3)}$, in terms of $H_{0}^{(1)}$.

The completion of the mathematical description of the problem requires an additional interfacial condition determine the shape of the interface between the fluids, which is the dynamical equilibrium boundary condition in which the surface traction suffers a discontinuity due to the surface tension:

$$
\left\|-p+2 \eta \frac{\partial v}{\partial y}+\mu\left(H_{n}^{2}-\frac{1}{2} H^{2}\right)\right\|_{j}^{j+1}=W_{j} \nabla \cdot \mathbf{n}_{j}
$$

These boundary conditions represented here are prescribed at the interface $y=\xi_{j}$. As the interface is deformed, all variables are slightly perturbed from their equilibrium values.

\subsection{Linear Perturbation and Solutions}

The analysis of linear theory, as presented in Chandrasekhar book [18], depends on neglecting the nonlinear terms from equation of motion as well as from the boundary conditions. The solution of the above system of governing equations and boundary conditions can be facilitated by defining a stream function, $\psi$ of the time and space coordinates, which automatically satisfies Equation (2), where

$$
u=\frac{\partial \psi}{\partial y}, \quad v=-\frac{\partial \psi}{\partial x}
$$

To solve the equations for the fluid phases under consideration, the two-dimensional finite disturbances are introduced into the equation of motion and continuity equation as well as the boundary conditions. As a customary in hydrodynamic stability analysis [18], where all quantities have exponential time and a spatial dependence and in view of a standard Fourier decomposition, we may assume that the solutions are in the form

$$
\begin{aligned}
& \xi_{j}(x, t)=\hat{\xi}_{j} \mathrm{e}^{i k x+\omega t}+c \cdot c \\
& \psi^{(r)}(x, y, t)=\hat{\psi}^{(r)}(y) \mathrm{e}^{i k x+\omega t}+c \cdot c \\
& \chi^{(r)}(x, y, t)=\hat{\chi}^{(r)}(y) \mathrm{e}^{i k x+\omega t}+c \cdot c \\
& p^{(r)}(x, y, t)=\hat{p}^{(r)}(y) \mathrm{e}^{i k x+\omega t}+c \cdot c
\end{aligned}
$$

where $\hat{\xi}_{l}$ is the initial amplitude of the disturbance, which is taken to be much smaller than the half-thickness $L$ of the middle sheet, $k$ is the wave number of the disturbance, which is assumed to be real and positive, $\omega$ is a complex frequency $\left(\omega=\omega_{r}+i \omega_{i}\right.$, where $\omega_{r}$ represents the rate of growth of the disturbance, $\omega_{i}$ is $2 \pi$ times the disturbance frequency), the symbol $i$ denotes $\sqrt{-1}$, the imaginary number and $c \cdot c$ represents the complex conjugate of the preceding terms.

Eliminating the pressure term from Equations (1) and (2) and using (15) and (17), we obtain the following equation

$$
\frac{\mathrm{d}^{4} \hat{\psi}^{(r)}}{\mathrm{d} y^{4}}-\left(\ell_{r}^{2}+k^{2}\right) \frac{\mathrm{d}^{2} \hat{\psi}^{(r)}}{\mathrm{d} y^{2}}+k^{2} \ell_{r}^{2} \hat{\psi}^{(r)}=0
$$

and

$$
\ell_{r}^{2}=k^{2}+\frac{\hat{\rho}^{(r)} R_{e} \omega}{\hat{\eta}^{(r)}}+\frac{1}{Q^{(r)}}
$$


Using the normal mode solution (17) we can obtain the pressure from Equation (1):

$$
p^{(r)}=\frac{1}{i k}\left\{\frac{\hat{\eta}^{(r)}}{R_{e}}\left[\frac{\partial^{3} \psi^{(r)}}{\partial y^{3}}+\frac{\partial^{3} \psi^{(r)}}{\partial x^{2} \partial y}-Q^{(r)-1} \frac{\partial \psi^{(r)}}{\partial y}\right]-\hat{\rho}^{(r)} \frac{\partial^{2} \psi^{(r)}}{\partial x \partial t}\right\}
$$

Substituting, the solution of the analytical solution of Equation (18) into Equation (15) we get

$$
\psi^{(r)}=\left(A_{1 r} \mathrm{e}^{k y}+A_{2 r} \mathrm{e}^{-k y}+A_{3 r} \mathrm{e}^{\ell_{r} y}+A_{4 r} \mathrm{e}^{-\ell_{r} y}\right) \mathrm{e}^{i k x+\omega t}+c \cdot c
$$

Also, the solution of the magnetic potential, in view of Equation (5) may be taken the form

$$
\chi^{(r)}=\left(B_{1 r} \mathrm{e}^{k y}+B_{2 r} \mathrm{e}^{-k y}\right) \mathrm{e}^{i k x+\omega t}+c \cdot c
$$

Since the disturbances vanish as $y \rightarrow \pm \infty$, this required that $A_{21}=A_{41}=A_{13}=A_{33}=0$ and $B_{21}=B_{13}=0$.

\section{Results and Discussion}

\subsection{Derivation of the Characteristic Equation}

In this section, we will derive the dispersion relation controlling the stability behavior of the system. When the obtained solutions of the stream function, magnetic potential and surface tension are inserted into Equations (6)(12), we have a linear homogeneous system of algebraic equations of the fourteen unknown coefficients $A_{p j}$, $B_{l j}, \quad \hat{\xi}_{l}, \quad(p=1,2,3,4)$. These homogeneous system of equations can be expressed in matrix form as

$$
\mathbf{M Z}=\mathbf{0}
$$

where $\mathbf{0}$ is a null vector, $\mathbf{Z}$ is a vector of unknown coefficients defined as

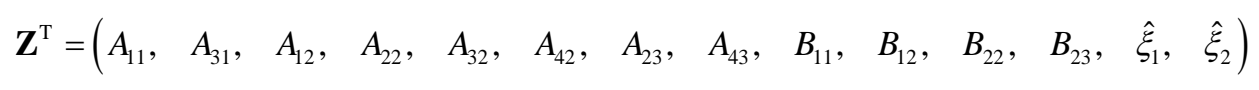

where the superscript $\mathrm{T}$ indicates the matrix transpose. A non-trivial solutions of the unknown coefficients $A_{p r}$, $B_{l r}, \quad \hat{\xi}_{j}$, exists if and only if the determinant of the $14 \times 14$ matrix $\mathbf{M}$ must be equal to zero, which yields a dispersion relation between the wave number $k$ and the perturbation frequency $\omega$ for specified values of other parameters, given by

$$
D\left(\omega, k ; R_{e}, H_{0}^{1}, \hat{\eta}^{(r)}, Q^{(r)}, W_{j}, \hat{\rho}^{(r)}, \hat{\mu}^{(r)}\right)=0
$$

which represents the linear dispersion equation for surface waves propagating through a viscous layer embedded between two other fluids with the influence of constant horizontal magnetic field. This dispersion relation controls the stability in the present problem. That is, each negative of the real part of $\omega$ corresponds to a stable mode of the interfacial disturbance. On the other hand, if the real part of $\omega$ is positive, the disturbance will grow in time and the flow becomes unstable.

It is clear that the eigenvalue relation (24) is somewhat more general and quite complex, since $\ell_{r}$ involves square roots and so one can obtain other characteristic relation as limiting cases. For an inviscid fluid we get the characteristic equation as special case from Equation (24) when $\hat{\eta}^{(r)}=0$. Thus by collecting the real and the imaginary terms in power order of $\omega$ with the help of symbolic computation software Mathematica, Equation (24) can be transformed into a polynomial algebraic equation of fourth order in the frequency $\omega$. Zakaria et al. [7] obtained a similar equation in their study of temporal stability of an inviscid fluids in porous media. Also, in the special case when the effect of the magnetic forces is absent and for the fluids flow through no-porous media, we get $\ell_{r}=\sqrt{k^{2}+\frac{\hat{\rho}^{(r)} R_{e} \omega}{\hat{\eta}^{(r)}}}$ and in this case the dispersion relation (28) is reduced to a non-polynomial algebraic equation for the frequency $\omega$ which coincides with that obtained by Kwak and Pozrikidis [19]. Another case is the limiting case of one interface between two continuum layers (non-porous medium), in which highly viscous fluids are considered. Thus we obtain a polynomial equation of fifth order in $\omega$, which is obtained before by Kumar and Singh [15] and Sunil et al. [20]. 
In the following, numerical applications are carried out to demonstrate the effects of various physical parameters on the stability criteria of the system. In the present work, we will numerically solve the implicit dispersion relation by means of the Chebyshev spectral tau method [21].

\subsection{Numerical Estimation for Stability Configuration}

In this section, the goal is to determine the numerical assess for the stability pictures for surface waves propagating through porous media. In order to present this examination, Equation (24) is used to control the stability behavior, which requires specification of the parameters: the magnetic field, the magnetic permeability, the porosity effect, the density, the viscosity. In the calculations given below all the physical parameters are sought in the dimensionless form as defined above. The stability of fluid sheets corresponds to negative values of the disturbance growth rate (i.e. $\omega_{r}<0$ ), and the disturbance growth rates of different fluids can be gained through solving the above corresponding dispersion relation numerically.

To show the effect of changes of the magnetic permeability ratio $\hat{\mu}^{(3)}\left(=\mu^{(3)} / \mu^{(2)}\right)$ on the stability behavior, the results for the calculations are displayed in Figure 2, partitions (a), (b) and (c) indicate the plane $\left(\omega_{r}-k\right)$. The graph displayed in this plane is evaluated for a system having the parameters given in the caption of Figure 2 , while the magnetic permeability $\hat{\mu}^{(3)}$ has some variation for the sake of comparison. In the graph 2 the values $0.5,1.5$ and 1.5 are selected for $\hat{\mu}^{(3)}$ correspond to the continuous, dashed and dotted curves respectively. Before, we discuss the stability of this graph we firstly define the critical wave number (also called the cutoff wave number) as given in [10] the value of the wave number at the point where the growth rate curve crosses the wave number axis in the plots of wave growth rate versus wave number. In other words the critical wave number is the value of the wave number, which separates the stable motions from the unstable ones and conversely, and can be obtained from the corresponding dispersion relations by setting $\omega_{r}=0$. It is apparent from the inspection of Figure 2, under the influence of the magnetic permeability $\hat{\mu}^{(3)}$, the growth rates with different magnetic permeability ratio keep almost identical for the wave numbers less than 0.4 , but increase

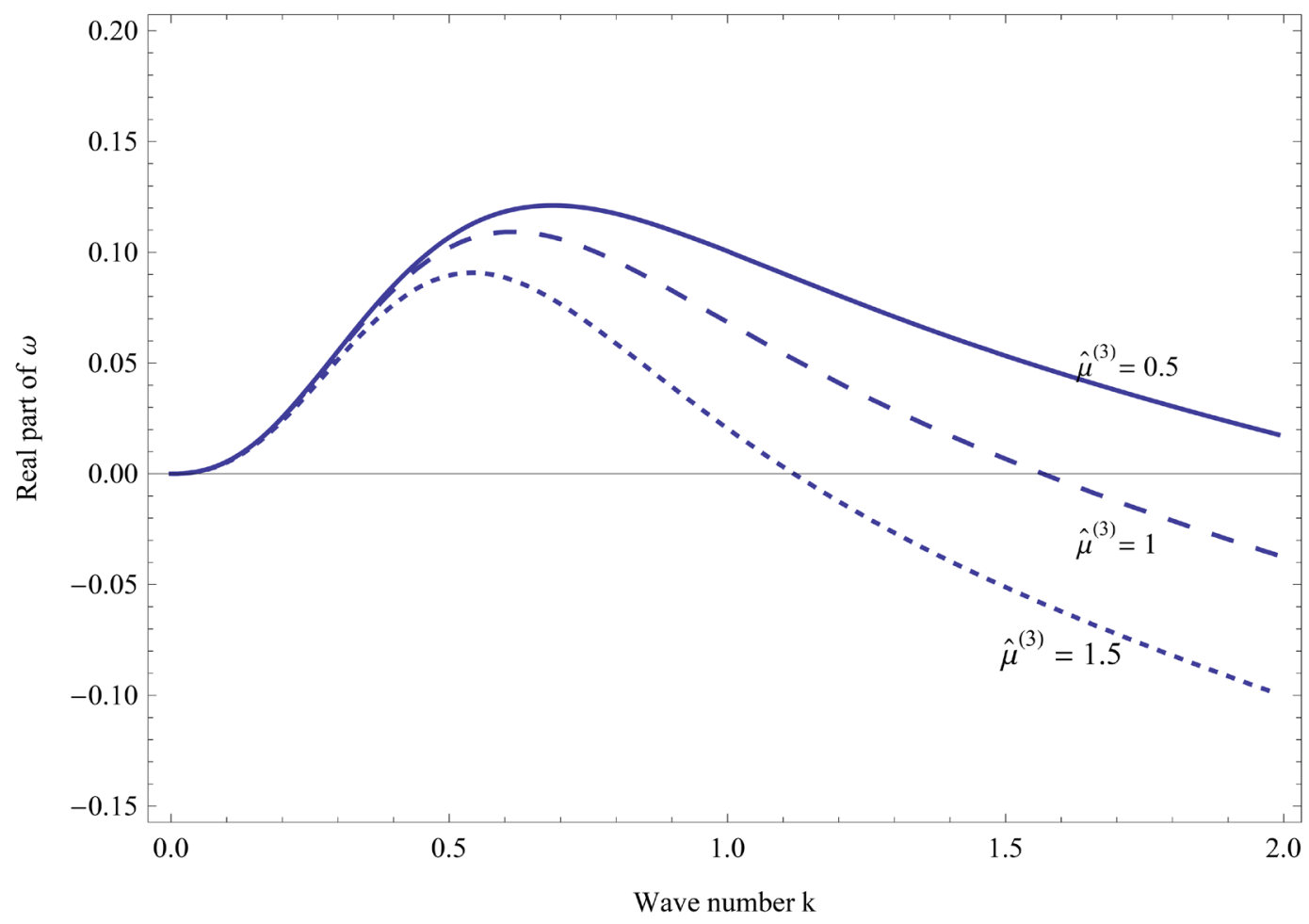

Figure 2. Influence of the magnetic permeability $\hat{\mu}^{(3)}$ in the plane $\left(\omega_{r}-k\right)$ at $H_{0}^{(1)}=0.5, \hat{\mu}^{(1)}=1.2$, $\hat{\rho}^{(1)}=0.6, \quad \hat{\rho}^{(3)}=0.8, \quad R_{e}=0.9, \quad \hat{\eta}^{(1)}=0.5, \quad \hat{\eta}^{(3)}=0.7, \quad Q^{(1)}=0.3, \quad Q^{(2)}=0.8, \quad Q^{(3)}=0.1, \quad W_{1}=3$, $W_{2}=2$ on the wave growth rate, with $\hat{\mu}^{(3)}=0.5$ (solid), 1 (dashed), 1.5 (dotted). 
correspondingly at higher values of the wave number, further the plane $\left(\omega_{r}-k\right)$ is divided into two regions. The first is $0<k<0.11$, which represent a stabilizing effect for increasing the parameter $\hat{\mu}^{(3)}$. The second region lie in the range $0.11<k<1.5$, since in this range, we notice that, when the magnetic permeability is increased, both the growth rates and the cutoff wave numbers of fluid sheets decrease. A general conclusion of the graph 2 reveals that, the stabilizing influence is found for increasing magnetic permeability ratio.

The examination of the influence of the magnetic field $H_{0}^{(1)}$ on the stability picture is illustrated in Figure 3 , for the same system considered in Figure 2. The results for calculations are displayed in the plane $\left(\omega_{r}-k\right)$. Since the stability arises according to the negative sign of the real part of the complex frequency $\omega$. Thus when the wave number is over the cutoff wave number, the fluid sheet is unstable. In this figure the solid curve is plotted at the value $H_{0}^{(1)}=1$, and the value $H_{0}^{(1)}=2$ corresponds to the dashed line, while the dotted curve represents the value $H_{0}^{(1)}=3$. The inspection of Figure 3 indicates that as the magnetic field is increased the growth rates increased, on other meaning the stable regions under the curves are decreased. Therefore, it is concluded that the magnetic field effects has destabilizing influence in the fluid sheets.

The examination of change of the lower to the middle fluid viscosity ratio $\hat{\eta}^{(1)}$ in the stability criteria is illustrated in Figure 4, where the values 0.2, 2.5 and 3 are choosing for the quantity $\hat{\eta}^{(1)}$. It is obvious from this graph, for every value of the $\hat{\eta}^{(1)}$, the corresponding curve crosses the wave number axis at three points (the cutoff wave numbers) and formed areas of stability and instability regions. Inspection of Figure 4 revels that the increasing of the viscosity ratio $\hat{\eta}^{(1)}$ tends to a reduction in the width of the unstable regions, whereas the stability region extended under the influence of the increasing $\hat{\eta}^{(1)}$. It is clear that from Figure 4 the viscosity ratio $\hat{\eta}^{(1)}$ has a stabilizing influence on the stability of the movement of the waves. Ozen et al. [22] have been obtained a similar conclusion in their studies of electrohydrodynamic linear stability of two immiscible fluids in channel flow. In Figure 5, in which the real part of the frequency $\omega$ is plotted against the wave number, the Reynolds number $R_{e}$ has the values $0.1,0.3$ and 0.7 to show its effect on the stability picture. Having checked the stability diagrams of this figure, it is discovered that the increasing of the Reynolds number leads to a contraction in the stability areas under the wave number axis, and consequently the Reynolds number has a destabilizing role on the stability behavior. Similar results were reported by Liu et al. [10] in their studies of the instability of two-dimensional non-Newtonian liquid sheets.

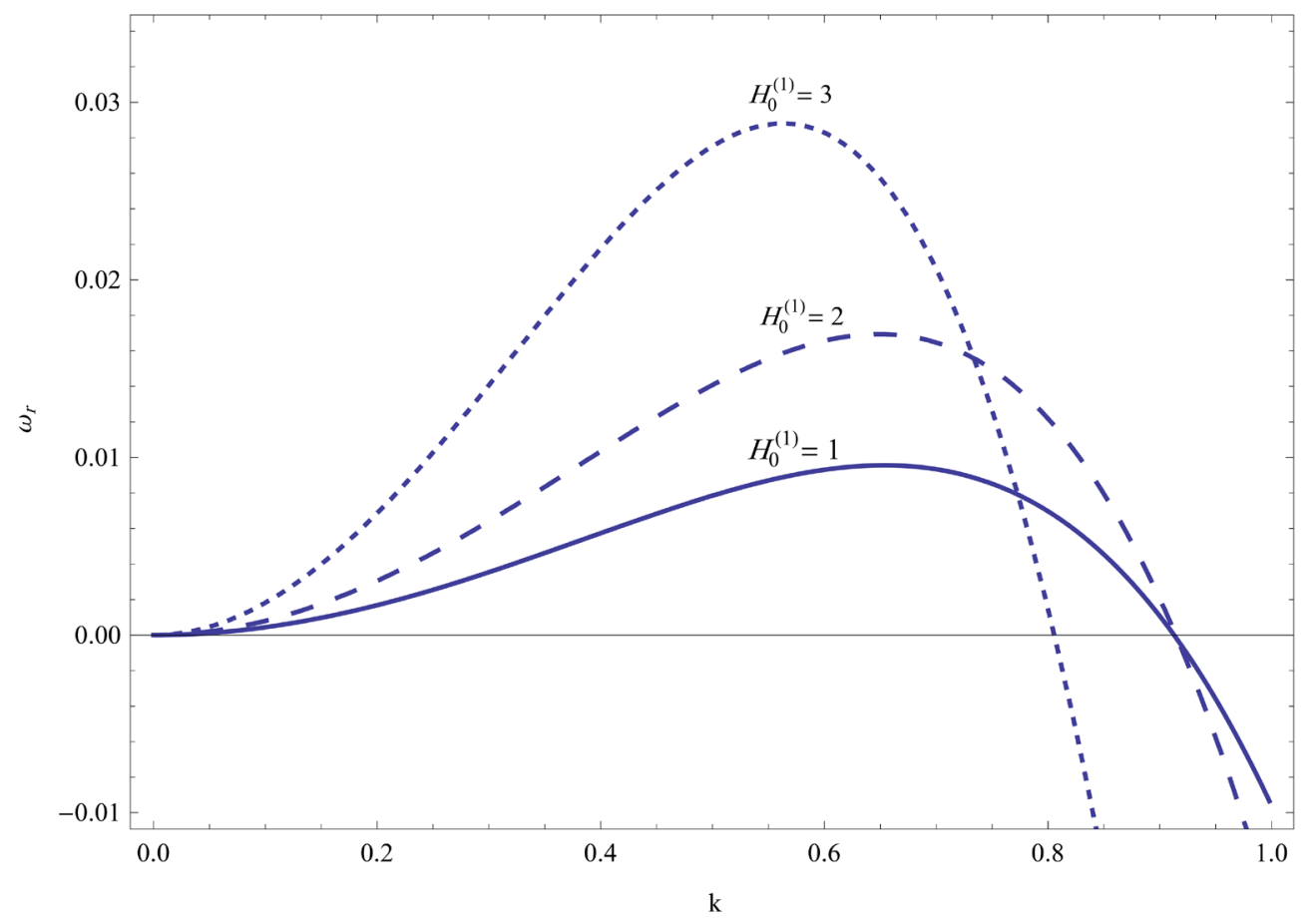

Figure 3. Effects of the magnetic field $H_{0}^{(1)}$ in the plane $\left(\omega_{r}-k\right)$, on the wave growth rate with $H_{0}^{(1)}=1,2,3$ at the same system given in Figure 2 . 


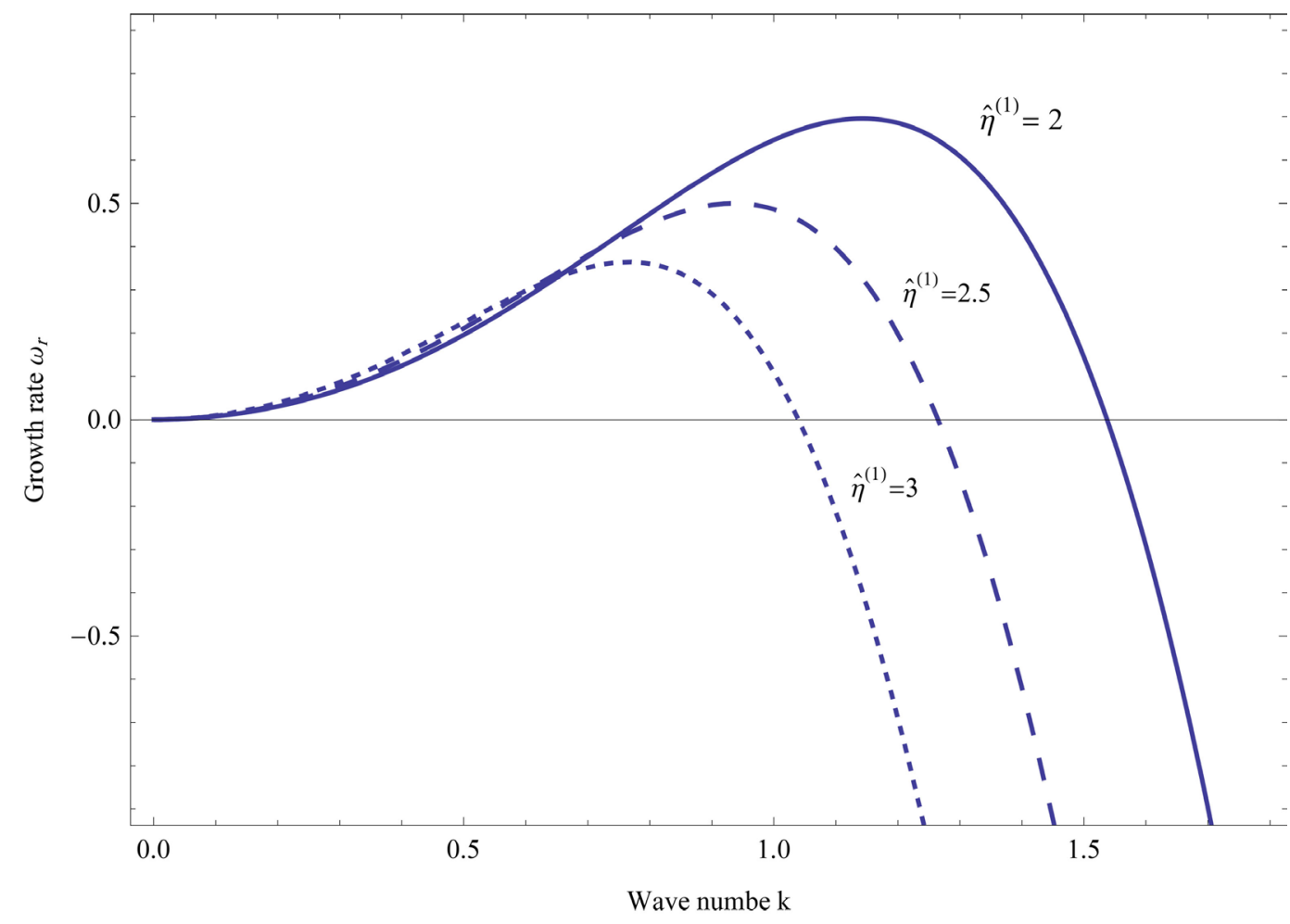

Figure 4. The graph is constructed for $\omega_{r}$ versus $k$, with $\hat{\eta}^{(1)}=2,2.5,3$.

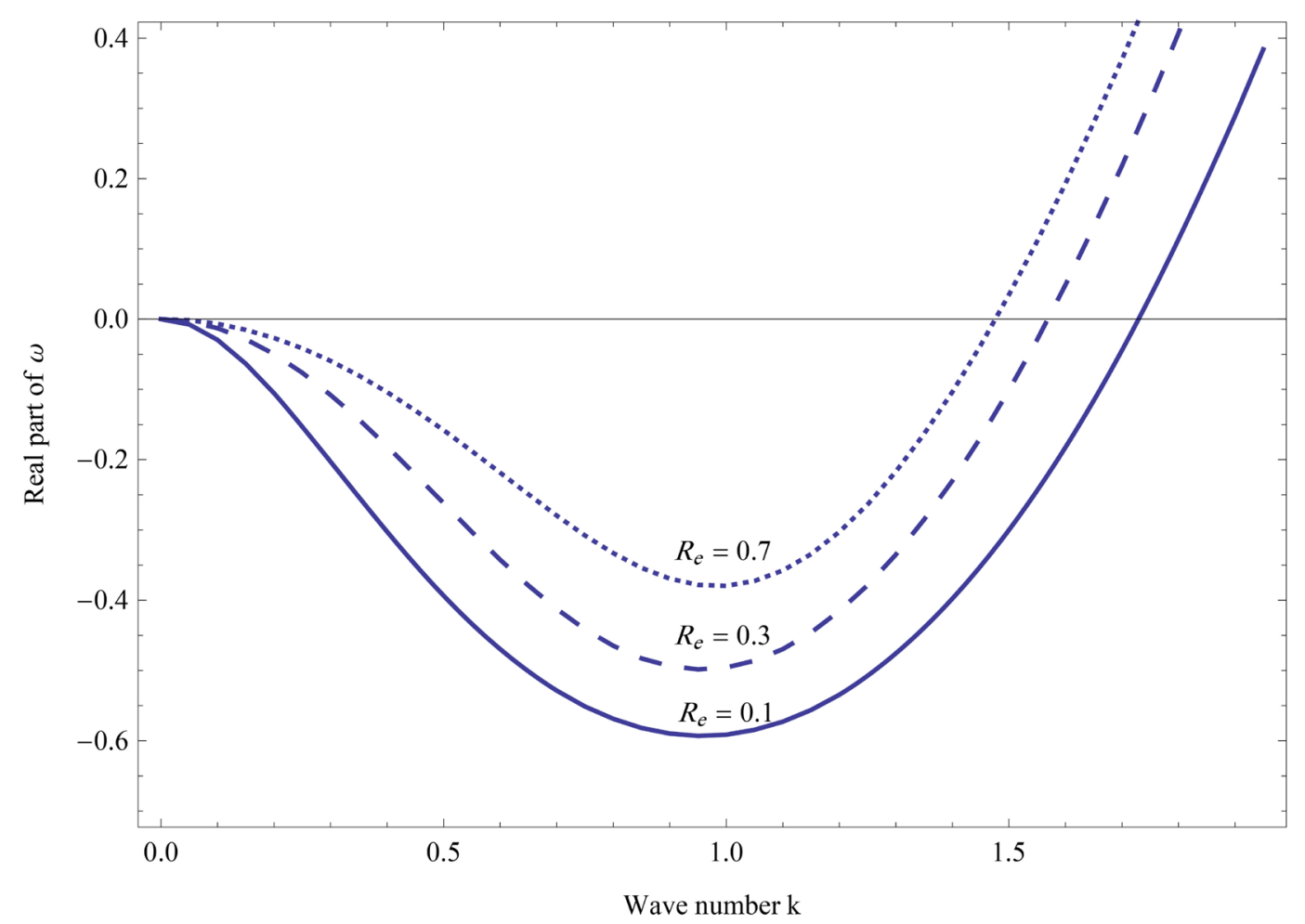

Figure 5. Represents the stability diagrams in the plane $\left(\omega_{r}-k\right)$ at the Reynolds number $R_{e}=0.1$ (solid), 0.3 (dashed), 0.7 (dotted). 
Figure 6 exhibits the effects of the the permeability parameter $Q^{(2)}$ on the stability behavior of the fluid layers. In this graph the solid, dashed and dotted curves represent the values $0.2,0.4$ and 0.9 in the plane $\left(\omega_{r}-R_{e}\right)$ of the parameter $Q^{(2)}$ respectively. Having noted the stability chart of this diagram, it is observed that the increasing of the permeability in the range $0<R_{e}<0.7$, leads to an contraction in the width of the instability regions (the regions under the curves and above the wave number axis correspond to the positive sing of the disturbance growth rate). On the other hand, through the interval $0.7<R_{e}<2$ the growth rates of instabilities with different $Q^{(2)}$ are increased. In general view of the graph 6, it is noticed that there are two roles of the variation of the the porous parameter $Q^{(2)}$, the first one is a stabilizing when the Reynolds number $R_{e}$ less than the value 0.7 , and the other role is a destabilizing when $R_{e}$ lies between the values 0.7 and 2. Hence the phenomenon of the dual (irregular) role is found for increasing the permeability parameter $Q^{(2)}$.

The influence of magnetic field $H_{0}^{(1)}$ on the flow behavior in terms of streamlines field is discussed through the parts of Figure 7 (a curve formed by the velocity vectors of each fluid particle at a certain time is called a streamline, in which the tangent at each point of this curve indicates the direction of fluid at that point). The streamlines in the physical domain are thus mapped into horizontal grid lines in the computational plane, thus resulting in a rectangular computational region. The streamlines show to be very effective tools to visualize a qualitative impression of the flow behavior during the motion. In the parts (a-c) of Figure 7, the streamlines picture is achieved by fixing the value of all the physical parameters as given in Figure 2, with $k=0.7$, $t=0.4, \quad \hat{\xi}_{1}=0.3$ and $\hat{\xi}_{2}=0.4$ where $H_{0}^{(1)}$ has three value for comparison. Snapshots of instantaneous streamlines of the stream function, are shown in Figure 7(a) at $H_{0}^{(1)}=0.8$. The inspection of this graph reveals that the flow consists of cells (contours) consisting of clockwise (positive values of streamlines) and anti clockwise (negative values of streamlines) circulations. In parts (b) and (c) of this graph, the values of $H_{0}^{(1)}$ are increased to 1.2 and 1.8 respectively.

A conclusion that may be made from the comparison among the parts (a-c) of Figure 7 is that the magnetic field leads to crowd in the concentration of the streamlines in the movement of the fluids. In other words, in the light of stability configuration, we notice that corresponding the parts (a-c) of Figure 7 there are three different values of the disturbance growth rate $\left(\omega_{r}\right)$, which are $0.049,0.66$ and 0.502 . Since the stability of fluid sheets arises to negative values of growth rate, thus it can be observed that the streamlines contours represent an unstable system. Hence the magnetic field has destabilizing influence, which coincide with the result given in Figure 3.

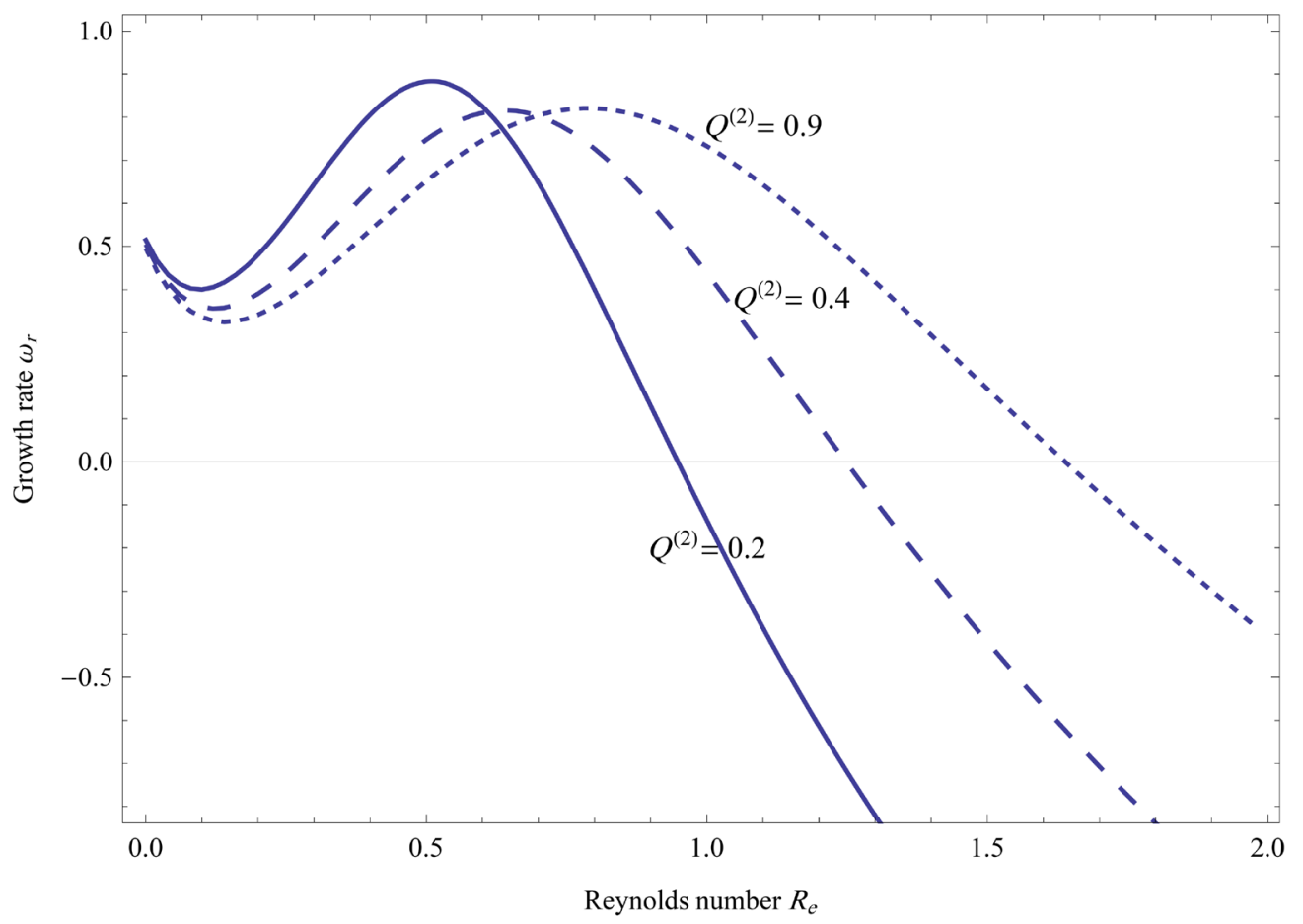

Figure 6. Illustrated in the plane $\left(\omega_{r}-R_{e}\right)$ with the permeability parameter $Q^{(2)}=0.2,0.4,0.9$. 
(a)

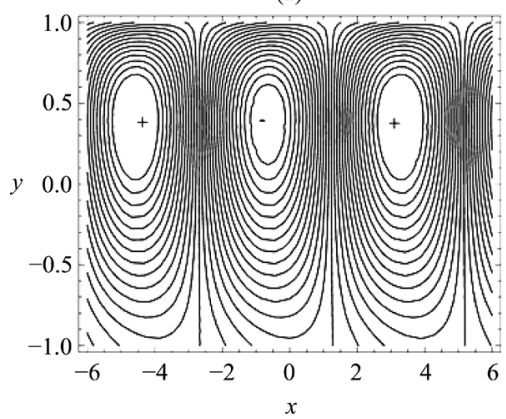

(b)

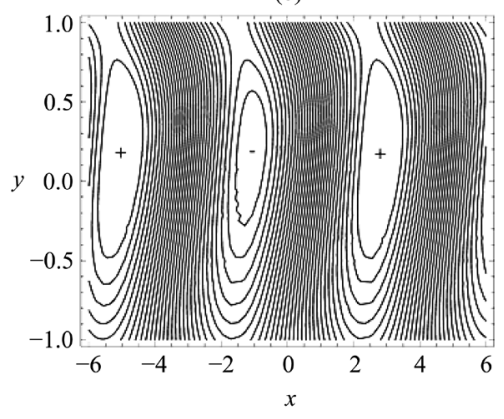

(c)

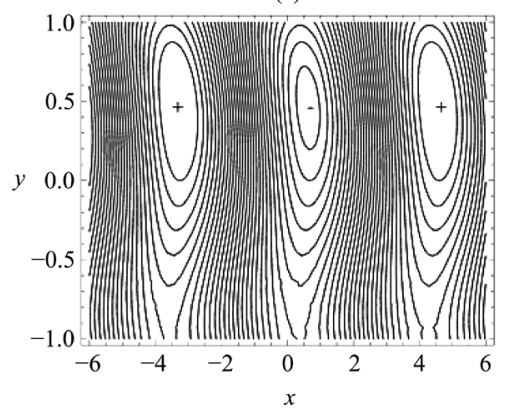

Figure 7. Streamlines contours for a system having the same parameters considered in Figure 2, with $k=0.7, t=0.4$, $\hat{\xi}_{1}=0.3$ and $\hat{\xi}_{2}=0.4$, where $H_{0}^{(1)}=0.8,1.2$ and 1.8 of the parts (a), (b), and (c), respectively.

\section{Conclusions}

This work is concerned with the influence of the normal magnetic field on the gravitational stability of a viscous fluid sheet of finite thickness. The sheet is embedded between two semi-infinite fluids layers moving in porous media, under the influence of magnetic field. The solutions of the linearized equations of motion under the boundary conditions lead to an implicit dispersion relation between the growth rate and wave number. The parameters governing the layers flow system, the magnetic properties and porosity effects strongly influence the wave forms and their amplitudes and hence the stability of the fluid. The stability criteria have been performed theoretically and numerically in which the physical parameters are put in the dimensionless form. Some stability diagrams have been plotted and discussed, in which the influence of the various parameters of the problem on the interface stability is thoroughly analyzed.

It has been found that the phenomenon of the dual (to be either stabilizing or destabilizing) role is found for increasing the permeability parameter. It is established that both the viscosity coefficient and the magnetic permeability damps the growth rate, introducing stabilizing influence, where a part of its kinetic energy may be absorbed. However, it is expected to be a more careful search would clarify that the motion of the interfacial waves will be more stable with the increase of the values of the viscosity as well as the magnetic permeability. In addition an increase of the lower to the middle fluid viscosity ratio decrease both the growth rate and the stability range of fluid sheet, which give a stabilizing influence on the stability behavior of the waves. This result confirmed the fact that when the lower fluid is more viscous than the upper, thus the system is stable. The role of the magnetic field and Reynolds number is to increase the amplitude of the disturbance leading to the destabilization state of the flow system, promote the oscillatory behavior.

\section{References}

[1] El-Dib, Y.O. (2003) Nonlinear Rayleigh? Taylor Instability for Hydromagnetic Darcian Flow: Effect of Free Surface Currents. Journal of Colloid and Interface Science, 259, 309-321. http://dx.doi.org/10.1016/S0021-9797(02)00208-4

[2] Bhatia, P.K. (1974) Rayleigh-Taylor Instability of Two Viscous Superposed Conducting Fluids. Nuovo Cimento, 19B, 161-168.

[3] Funada, T. and Joseph, D.D. (2001) Viscous Potential Flow Analysis of Kelvin-Helmholtz Instability in a Channel. Journal of Fluid Mechanics, 445, 263-283. http://dx.doi.org/10.1017/S0022112001005572

[4] Elhefnawy, A.F. (1995) Intervals of an Unsteady Electrohydrodynamic Kelvin-Helmoltz Stability. Physica A, 214, 229-241. http://dx.doi.org/10.1016/0378-4371(94)00232-I

[5] Drazin, P.G. and Reid, W.H. (1981) Hydrodynamic Stability. University Press, Cambridge.

[6] Joseph, D.D. (1976) Stability of Fluid Motions II. Springer-Verlag, New York.

[7] Zakaria, K., Sirwah, M.A. and Alkharashi, S. (2008) Temporal Stability of Superposed Magnetic Fluids in Porous Media. Physica Scripta, 77, 1-20. http://dx.doi.org/10.1088/0031-8949/77/02/025401

[8] Zakaria, K., Sirwah, M.A. and Alkharashi, S.A. (2012) Non-Linear Analysis of Creeping Flow on the Inclined Permeable Substrate Plane Subjected to an Electric Field. International Journal of Non-Linear Mechanics, 47, 577-598. http://dx.doi.org/10.1016/j.ijnonlinmec.2011.11.010 
[9] Wray, A.W., Papageorgiou, D.T. and Matar, O.K. (2013) Electrified Coating Flows on Vertical Fibres: Enhancement or Suppression of Interfacial Dynamics. Journal of Fluid Mechanics, 735, 427-456. http://dx.doi.org/10.1017/jfm.2013.505

[10] Liu, Z., Brenn, G. and Durst, F. (1998) Linear Analysis of the Instability of Two-Dimensional Non-Newtonian Liquid Sheets. Journal of Non-Newtonian Fluid Mechanics, 78, 133-166. http://dx.doi.org/10.1016/S0377-0257(98)00060-3

[11] Sirwah, M.A. (2012) Linear Instability of the Electrified Free Interface between Two Cylindrical Shells of Viscoelastic Fluids through Porous Media. Acta Mechanica Sinica, 28, 1572-1589. http://dx.doi.org/10.1007/s10409-012-0208-2

[12] Tan, W.C. and Masuoka, T. (2005) Stokes' First Problem for an Oldroyd-B Fluid in a Porous Half Space. Physics of Fluids, 17, Article ID: 023101. http://dx.doi.org/10.1063/1.1850409

[13] Zakaria, K. (2012) Long Interfacial Waves inside an Inclined Permeable Channel. International Journal of Non-Linear Mechanics, 47, 42-48. http://dx.doi.org/10.1016/j.ijnonlinmec.2012.02.002

[14] Khan, M., Saleem, M., Fetecau, C. and Hayat, T. (2007) Transient Oscillatory and Constantly Accelerated Non-Newtonian Flow in a Porous Medium. International Journal of Non-Linear Mechanics, 42, 1224-1239. http://dx.doi.org/10.1016/j.ijnonlinmec.2007.09.008

[15] Kumar, P. and Singh, G.J. (2006) Stability of Two Superposed Rivlin-Ericksen Viscoelastic Fluids in the Presence of Suspended Particles. Romanian Journal of Physics, 51, 927-935.

[16] Woodson, H.H. and Melcher, J.R. (1968) Electromechanical Dynamics. John Wiley and Sons, Hoboken.

[17] Rosensweig, R.E. (1985) Ferrohydrodynamics. Cambridge University Press, Cambridge.

[18] Chandrasekhar, S. (1961) Hydrodynamic and Hydromagnetic Stability. Oxford University Press, Oxford.

[19] Kwak, S. and Pozrikidis, C. (2001) Effect of Surfactants on the Instability of a Liquid Thread of Annular Layer. Part I: Quiescent Fluids. International Journal of Multiphase Flow, 27, 1-37. http://dx.doi.org/10.1016/S0301-9322(00)00011-2

[20] Sharma, R.C. and Chandel, R.S. (2002) On Superposed Couple-Stress Fluids in Porous Medium in Hydromagnetics. Zeitschrift für Naturforschung, 57a, 955-960.

[21] Boyd, J.P. (2001) Chebyshev and Fourier Spectral Methods. Dover Publications Inc., Mineola.

[22] Ozen, O., Aubry, N., Papageorgiou, D.T. and Petropoulos, P.G. (2006) Electrohydrodynamic Linear Stability of Two Immiscible Fluids in Channel Flow. Electrochimica Acta, 51, 5316-5323. http://dx.doi.org/10.1016/j.electacta.2006.02.002 